Proceedings of the European Conference "Physics of Magnetism '99", Poznań 1999

\title{
CORRELATION INDUCED OPTIMIZATION OF WAVE FUNCTIONS: THE HUBBARD CHAIN
}

\author{
J. SPalek, R. Podsiadey \\ Marian Smoluchowski Institute of Physics, Jagiellonian University \\ Reymonta 4, 30-059 Kraków, Poland \\ AND W. WóJcIK \\ Institute of Physics, Tadeusz Kościuszko Technical University \\ Podchorażych 1, 30-084 Kraków, Poland
}

\begin{abstract}
We determine the explicit form of the single-particle Wannier functions $\left\{w_{i}(r)\right\}$ appearing in the parameters of quantum models. The method is illustrated on the example of the Hubbard chain, for which we derive the renormalized wave equation starting from a variational principle and by treating the system ground state energy as a functional of $\left\{w_{i}(r)\right\}$ and their derivatives. In this manner, the optimized basis is obtained only after the electronic correlations have been included in the rigorous Lieb-Wu solution. The results for the ground state energy and the size of the renormalized $s$-type orbitals, both as a function of interatomic distance, are calculated explicitly.
\end{abstract}

PACS numbers: 71.10.Fd, 71.15.Fv, 31.25.Nj

\section{Introduction}

The physics of correlated electronic systems is customarily discussed starting from a parametrized model in the second quantization representation. The examples are the Hubbard, $t-J$, Kondo, and Anderson (both impurity and lattice versions) models. In all these models the entities such as the hopping integral $t$ (or hybridization $V$ ), the intraatomic Coulomb $U$, and the nearest-neighbor Coulomb $(K)$ and exchange $(J)$ integrals are taken as parameters containing single-particle Wannier functions $\left\{w_{i}(r)\right\}$. The solution is analysed in terms of these parameters.

The Lieb-Wu solution [1] for the Hubbard chain has among others one nontrivial property, namely the metallic state is not stable even for very small magnitude of $U$. Therefore, it is legitimate to ask whether one should not readjust the single-particle basis $\left\{w_{i}(r)\right\}$ to the situation when the interaction plays such a crucial role in determining the character of the quantum ground state. In this contribution we propose a renormalized wave equation determining those function by regarding the rigorous expression for the ground state energy [1] as a functional of the functions $\left\{w_{i}(r)\right\}$ and their derivatives under the constraint that the basis $\left\{w_{i}(r)\right\}$ is orthonormal. Variational solution of this extra equation allows for determination of all quantities as a function of the interatomic distance. We present here only the main points of the approach and provide basic results; the interested reader is referred to the forthcoming full version of the paper [2]. 


\section{Optimized orbitals of $s$-type}

The ground-state-energy expression obtained by Lieb and Wu [1] for the Hubbard chain composed of $N$ atoms and $N_{\mathrm{e}}=N$ electrons, when written down in physical units, has the form

$$
\frac{E_{G}}{N}=E_{0}+4 t \int_{0}^{\infty} \mathrm{d} \omega \frac{J_{0}(\omega) J_{1}(\omega)}{\omega[1+\exp (-\omega U / 2 t)]}
$$

where $E_{0}$ contains both the atomic $\left(\epsilon_{\mathrm{a}}\right)$ and the lattice $\left(\epsilon_{\text {latt }}\right)$ contributions*, $t$ is the nearest neighbor hopping integral, $U$ is the intraatomic (Hubbard) part of the Coulomb interaction between electrons, and $J_{n}(x)$ is the Bessel function with $n=0$ or 1 . The last two parameters are defined as $t=\left\langle w_{i}\left|-\frac{\hbar^{2}}{2 m} \nabla^{2}+V(r)\right| w_{i \pm 1}\right\rangle \equiv$ $\left\langle w_{i}\left|H_{1}\right| w_{i \pm 1}\right\rangle$, and $U=\left\langle w_{i} w_{i}\left|\frac{e^{2}}{\left|r-r^{\prime}\right|}\right| w_{i} w_{i}\right\rangle \equiv\left\langle w_{i}^{2}\left|V_{12}\right| w_{i}^{2}\right\rangle$, where $V(\boldsymbol{r})$ is the periodic potential of electron (here taken in the tight-binding approximation). From the statement of the problem in the preceding section it follows that $E_{G}$ can be regarded as a functional of the basis $\left\{w_{i}(r)\right\}$, i.e. $E_{G} \equiv E_{G}\left\{w_{i}(\boldsymbol{r}), \nabla w_{i}(\boldsymbol{r})\right\}$. Therefore, the corresponding Lagrange-Euler equation can be derived under the constraint that each wave function $w_{i}(r)$ is normalized. Thus, the functional to be minimized is

$$
F\left\{w_{i}, \nabla w_{i}\right\} \equiv E_{G}\left\{w_{i}, \nabla w_{i}\right\}-\sum_{i} \lambda_{i}\left(\int \mathrm{d}^{d} r\left|w_{i}(r)\right|^{2}-1\right),
$$

and the renormalized wave equation for the function $w_{i}(r)$ has the general form ${ }^{\dagger}$

$$
\frac{\delta E_{G}}{\delta w_{i}}-\nabla \frac{\delta E_{G}}{\delta \nabla w_{i}}-\lambda_{i} w_{i}=0
$$

The renormalized Hamiltonian is defined through the relation $H_{\mathrm{R}} w_{i}=\lambda_{i} w_{i}$, where the eigenvalue $\lambda_{i}$ plays the role of the Lagrange multiplier in a constraint. This is quite complicated differential-integral and non-linear equation, which we analyze subsequently variationally in terms of readjusted (optimized) orbitals of $s$-type.

To find optimized orbitals we note that the Wannier function $w_{i}(r)$ can be expressed by atomic functions $\left\{\Psi_{i}(r)\right\}$ in the tight-binding approximation (TBA) in the form

$$
w_{i}(r)=\beta \Psi_{i}(r)+\gamma\left[\Psi_{i+1}(r)+\Psi_{i-1}(r)\right]
$$

where the coefficients are

$$
\beta=\frac{1+\sqrt{1-3 S^{2}}}{\sqrt{2-5 S^{2}+2\left(1-2 S^{2}\right) \sqrt{1-3 S^{2}}}}, \gamma=\frac{-S}{\sqrt{2-5 S^{2}+2\left(1-2 S^{2}\right) \sqrt{1-3 S^{2}}}}
$$

and $S=\left\langle\Psi_{i} \mid \Psi_{i \pm 1}\right\rangle$ is the overlap integral.

*The solution in Ref. [1] is performed for $\epsilon_{\mathrm{a}}=0, t=-1$. We need the explicit expressions for both of those quantities in $E_{G}$, as they contain the functions $w_{i}(r)$ and hence depend explicitly on the interatomic distance $R$.

tSuch procedure is composed of including the correlations in the Fock space and then readjusting the orbital adiabatically. It was utilized before in the context of a single electron coupled to thermodynamic fluctuations, cf. J. Spałek, Phys. Rev. B 30, 5345 (1984). 
In accordance with the Hubbard model definition, the atomic wave functions should be of the $s$-like form: $\Psi_{i}(r)=\left(\alpha^{3} / \pi\right)^{1 / 2} \exp \left(-\alpha\left|r-\boldsymbol{R}_{i}\right|\right)$ and hence Eq. (2) reduces to the optimization of the orbital size $\left(\alpha^{-1}\right)$. Additionally, we have to check a posteriori if the tight-binding approximation holds (i.e. $S$ is substantially smaller than unity). Note also that the definition of $w_{i}$ is essentially a definition of orthogonalized atomic basis. Therefore, one can express the parameters $\epsilon_{\mathrm{a}}, t$, and $U$ in terms of the corresponding (primed) quantities in the nonorthogonal (atomic) basis in the following manner:

$$
\begin{aligned}
& \epsilon_{\mathrm{a}}=\left(\beta^{2}+2 \gamma^{2}\right) \epsilon_{\mathrm{a}}^{\prime}+4 \beta \gamma t^{\prime} \\
& t=\left(\beta^{2}+3 \gamma^{2}\right) t^{\prime}+2 \beta \gamma \epsilon_{\mathrm{a}}^{\prime}
\end{aligned}
$$

and

$$
U=\left(\beta^{4}+2 \gamma^{4}\right) U^{\prime}+8 \beta^{3} \gamma V^{\prime}+8 \beta \gamma^{3} V^{\prime}+4 \beta^{2} \gamma^{2} K^{\prime}+8 \beta^{2} \gamma^{2} J^{\prime},
$$

where $\epsilon_{\mathrm{a}}^{\prime}=\left\langle\Psi_{i}\left|H_{1}\right| \Psi_{i}\right\rangle, t^{\prime}=\left\langle\Psi_{i}\left|H_{1}\right| \Psi_{i \pm 1}\right\rangle, V^{\prime}=\left\langle\Psi_{i}^{2}\left|V_{12}\right| \Psi_{i} \Psi_{i \pm 1}\right\rangle, \quad K^{\prime}=$ $\left\langle\Psi_{i}^{2}\left|V_{12}\right| \Psi_{i \pm 1}^{2}\right\rangle$, and $J^{\prime}=\left\langle\Psi_{i} \Psi_{i \pm 1}\left|V_{12}\right| \Psi_{i \pm 1} \Psi_{i}\right\rangle$. The primed quantities can be expressed analytically by the orbital size $\alpha^{-1}$ and the interatomic distance $R$, and have been tabulated by Slater [3].

The optimized value of the ground state energy as a function of the atomic distance $R$ (in units of the Bohr radius $a_{0} \approx 0.5 \AA$ ) is provided in Fig. 1 . We have also included the nearest-neighbor ion-ion repulsion $\epsilon_{\text {latt }}=2 e^{2} / R$ in $E_{G}$. The Hubbard chain is stable for any $R$. The inset displays the energy as a function of $U /|t|$. In the asymptotic regime $R>5 a_{0}, E_{G} \rightarrow-1 \mathrm{Ry}$, the atomic limit value per site. For comparison, we have plotted in Fig. 2 the orbital size $\left(\alpha^{-1} / a_{0}\right)$ and the overlap integral $S$ (in the inset), both as a function of $R / a_{0}$. The tight-binding scheme is applicable in the regime $R>3.5 a_{0} \approx 1.8 \AA$. The renormalization of the orbital size is small, but nonmonotonic.

The determination of the optimal orbital size allows for calculation of the microscopic parameters. In Fig. 3 we have shown the $R$ dependence of the ratio $|t| / U$. In the whole regime $W / U=4|t| / U<1$, i.e. the system is strongly correlated. Here it is achieved for interatomic distance $R \sim 7 a_{0} \approx 3.5 \AA$.

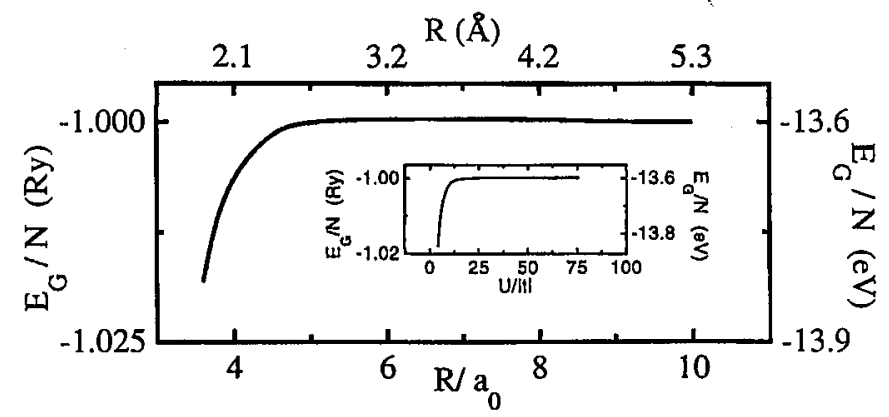

Fig. 1. Ground state energy $E_{G}$ of the Hubbard chain (per site) as a function of relative interatomic distance $R / a_{0}$. The atomic energy and the interionic Coulomb repulsion is included. The inset provides $E_{G}$ versus $U /|t|$. 

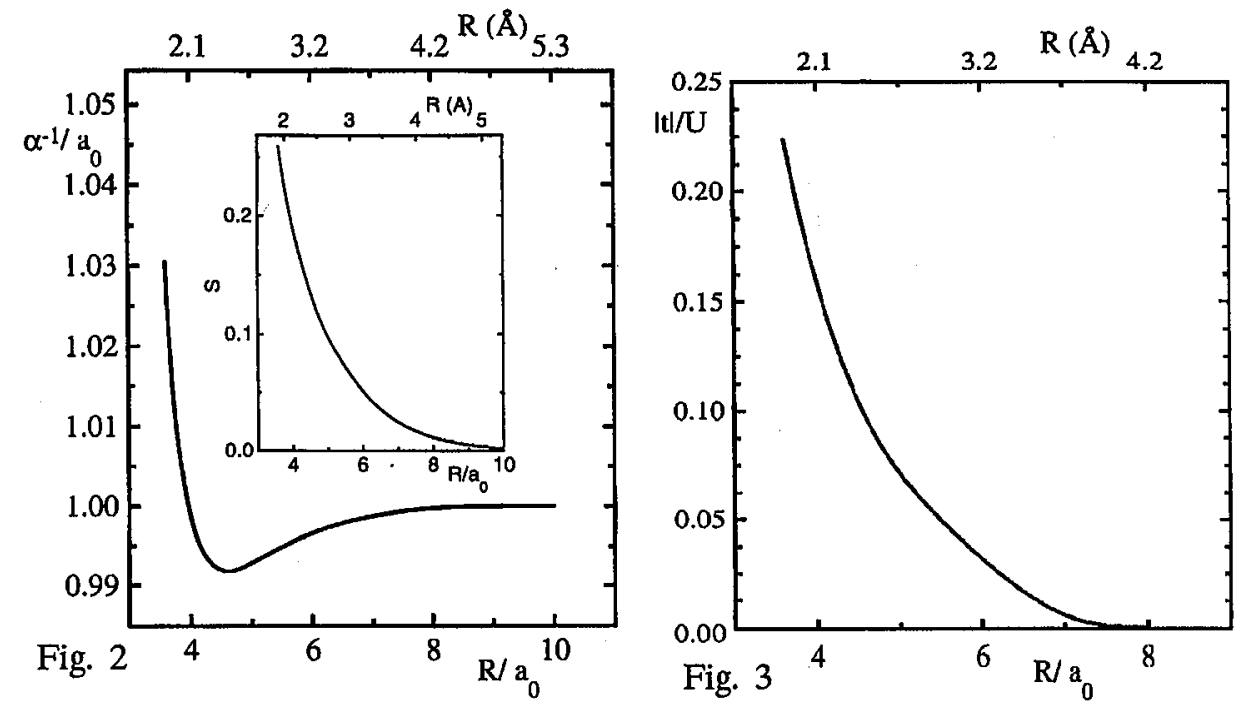

Fig. 2. Optimal size of the atomic $1 s$ orbit - in units of the atomic Bohr radius - as a function of interatomic distance. In the inset the overlap integral vs. $R$ is displayed. Fig. 3. $|t| / U$ ratio versus interatomic distance $R / A_{0}$.

\section{Concluding remarks}

In this paper we have proposed a novel method of single-particle wave function readjustment after the electron correlations have been treated carefully. The method was adopted to the Hubbard-chain case, where a well-known treatment of Lieb and Wu [1] provides a rigorous expression for the ground state energy. A similar approach is possible for the Anderson-impurity model [4]; this will be discussed separately.

One can say that proposed by us [2] wave-function readjustment closes the Hubbard chain solution in the sense that it determines the physical properties as a function of experimentally accessible interatomic distance. In this manner, one can discuss explicitly e.g. the pressure dependence of various quantities of interest.

The authors acknowledge the financial support through the Committee for Scientific Research grant No. 2P03B 12912.

\section{References}

[1] E.H. Lieb, F.Y. Wu, Phys. Rev. Lett. 20, 1445 (1968).

[2] J. Spałek, R. Podsiadły, W. Wójcik, submitted to Phys. Rev. B.

[3] J.C. Slater, Quantum Theory of Molecules and Solids, Vol. 1, McGraw-Hill, New York 1963, p. 50ff.

[4] N. Kawakami, A. Okiji, Phys. Lett. A 86, 483 (1981). 